Osteo arthritis, aetiology, pathology and animal models.

\begin{tabular}{l|l}
\hline POS0371 & BIOLOGICAL REPRODUCIBILITY OF TARGETED \\
LIPIDOME ANALYSES IN PLASMA AND \\
ERYTHROCYTES OVER A 6-WEEK PERIOD
\end{tabular}

M. Loef ${ }^{1}$, J. von Hegedus ${ }^{1}$, M. Ghorasaini ${ }^{2}$, F. Kroon ${ }^{1}$, M. Giera ${ }^{2}$, A. IoanFacsinay ${ }^{1}$, M. Kloppenburg ${ }^{1,3} .{ }^{1}$ Leiden University Medical Center (LUMC), Rheumatology, Leiden, Netherlands; ${ }^{2}$ Leiden University Medical Center, Center for Proteomics and Metabolomics, Leiden, Netherlands; ${ }^{3}$ Leiden University Medical Center, Clinical Epidemiology, Leiden, Netherlands

Background: Lipidomics analysis has become a valuable technology for understanding patho-physiological mechanisms and the identification of candidate biomarkers in rheumatic musculoskeletal disorders. Variability in within-subject repeated measurements may lead to bias towards the null when estimating the association between biomarkers and a disease or treatment. Hence, information regarding the stability of the metabolite levels over time is essential.

Objectives: We aimed to assess the lipid composition and biological reproducibility of lipid measurements in plasma and erythrocytes.

Methods: Plasma and erythrocyte samples from 42 osteoarthritis patients $(77 \%$ women, mean age 65 years, mean BMI $27 \mathrm{~kg} / \mathrm{m} 2$ ), obtained non-fasted at baseline and six weeks, were used for the quantitative measurement of up to 1000 lipid species across 13 lipid classes with the LipidyzerTM platform in nmol/mL. Data was processed based on the relative standard deviation of quality controls, taking batch effects into account. Intraclass correlation coefficients (ICCs) and corresponding $95 \%$ confidence intervals $(\mathrm{Cl})$ were calculated to investigate the variability of the lipid concentrations between timepoints. The ICC distribution of lipid metabolites in plasma and erythrocytes were compared using two-sided paired Wilcoxon tests. Results: We measured 778 lipids in plasma, compared to 916 lipids in erythrocytes. After data processing, the analyses included 630 lipids in plasma, and 286 in erythrocytes. From these, 243 lipids overlapped between sample types. Major differences were observed between the sample types in the number of lipids per lipid class and the total concentration of the lipids within a class. Triacylglycerols (TAG) and cholesteryl esters (CE) were more abundant in plasma. Conversely, phosphatidylethanolamines (PE), sphingomyelins (SM) and ceramides (CER) were less abundant in plasma compared to erythrocytes (table 1). In plasma $78 \%$ of lipid measurements were good to excellently reproduced, with an overall median ICC 0.69. Compared to plasma, a considerably lower amount (35\%) of lipids were well reproduced in erythrocytes. Median reproducibility of lipids in erythrocytes was 0.51 . Figure 1 shows the ICC score distribution in plasma with erythrocytes, with a significantly better reproducibility in plasma $(p$-value $<0.001)$. However, while overall reproducibility was better in plasma, this was not observed for all lipid classes. At class-level, reproducibility in plasma was superior for TAGs and CEs, while CERs, DAGs, (L)PEs and SMs showed better reproducibility in erythrocytes.

Conclusion: In plasma biological reproducibility was good for most lipid measurements. Although overall reproducibility was better in plasma compared to erythrocytes, notable differences were observed at individual- and lipid classlevel that may favour the use of a particular sample type.

Table 1. Number of individual lipids per class and class concentrations in plasma and erythrocytes

\begin{tabular}{|c|c|c|c|c|}
\hline & \multicolumn{2}{|r|}{ Plasma } & \multicolumn{2}{|r|}{ Erythrocytes } \\
\hline & $\begin{array}{l}\text { Number } \\
\text { of lipid } \\
\text { species }\end{array}$ & $\begin{array}{c}\text { Class } \\
\text { concentration } \\
(\mathrm{nmol} / \mathrm{mL})\end{array}$ & $\begin{array}{l}\text { Number } \\
\text { of lipid } \\
\text { species }\end{array}$ & $\begin{array}{c}\text { Class } \\
\text { concentration } \\
(\mathrm{nmol} / \mathrm{mL})\end{array}$ \\
\hline Triacylglycerols & 482 & $\begin{array}{c}1579.4 \\
(1064.9-3195.2)\end{array}$ & 134 & $6.5(5.6-9.4)$ \\
\hline Diacylglycerols & 9 & $13.3(8.4-22.2)$ & 10 & $5.8(4.7-6.2)$ \\
\hline Free fatty acids & 20 & $745.3(552.0-1202.9)$ & 20 & $486.9(379.2-669.2)$ \\
\hline Cholesteryl esters & 24 & $4571.6(4065.1-5521.3)$ & 5 & $1.2(0.9-1.7)$ \\
\hline Phosphatidylcholines & 31 & 4013.7 (3203.1-4661.6) & 42 & $\begin{array}{c}3899.2 \\
(3723.0-4296.6)\end{array}$ \\
\hline Phosphatidylethanolamines & 26 & $156.2(120.9-180.3)$ & 42 & $\begin{array}{c}3954.6 \\
(3721.9-4323.3)\end{array}$ \\
\hline Lysophosphatidylcholines & 9 & 385.9 (335.6-442.9) & 7 & $119.8(109.7-168.9)$ \\
\hline Lysophosphatidylethanolamines & 2 & $4.2(3.5-4.9)$ & 4 & $8.6(6.8-9.7)$ \\
\hline Sphingomyelins & 12 & $\begin{array}{c}1204.6 \\
(1037.0-1351.9)\end{array}$ & 8 & $\begin{array}{c}2695.8 \\
(2434.8-2815.6)\end{array}$ \\
\hline Ceramides & 6 & $14.1(11.9-17.4)$ & 7 & $163.0(133.3-186.4)$ \\
\hline Dihydroceramides & 2 & $1.0(0.8-1.3)$ & 1 & $1.8(1.4-2.1)$ \\
\hline Hexosylceramides & 5 & $5.1(4.7-5.9)$ & 4 & $5.6(5.0-7.4)$ \\
\hline Lactosylceramides & 2 & $3.4(2.7-3.8)$ & 2 & $23.8(20.6-33.5)$ \\
\hline
\end{tabular}

Numbers represent median (interquartile range) unless otherwise specified. Data represents baseline measurements.

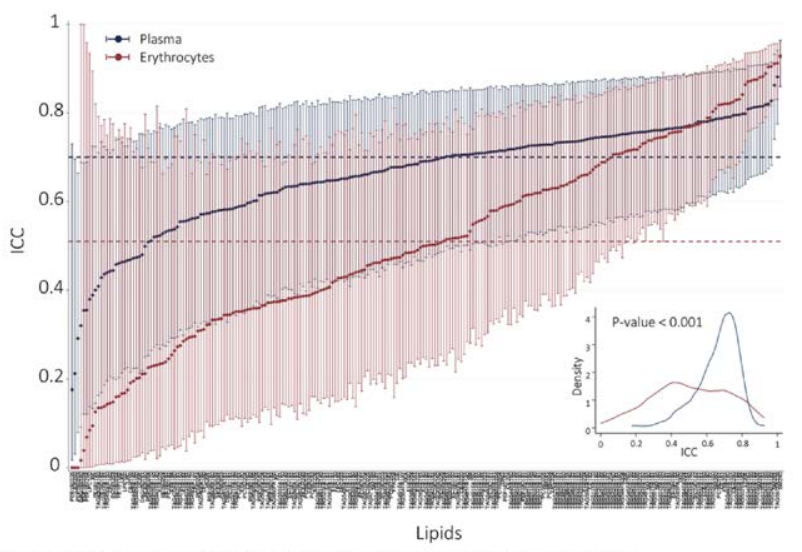

Figure 1. The ICC score distribution of lipids measured in plasma and erythrocytes.

Lipids are ranked ascending by plasma samples. A density plot of the ICC score distributions from plasma and erythrocyte samples is inserted in the lower right corner; the p-value represents the result from the Wilcoxon signed-rank test used to compare the distributions. The dashed lines correspond to the median ICC scores of lipids in plasma (blue) and erythrocytes (red).

Disclosure of Interests: Marieke Loef: None declared, Johannes von Hegedus: None declared, Mohan Ghorasaini: None declared, Féline Kroon: None declared, Martin Giera Shareholder of: Pfizer, Consultant of: Boehringer Ingelheim Pharma, Andreea loan-Facsinay: None declared, Margreet Kloppenburg: None declared

DOI: 10.1136/annrheumdis-2021-eular.20

\section{POS0372 LORECIVIVINT (SM04690), AN INTRA-ARTICULAR, SMALL-MOLECULE CLK2/DYRK1A INHIBITOR THAT MODULATES THE WNT PATHWAY, AS A POTENTIAL TREATMENT FOR MENISCAL INJURIES}

T. Seo ${ }^{1}$, V. Deshmukh ${ }^{1}$, Y. Yazici' ${ }^{2}{ }^{1}$ Samumed, LLC, Biology, San Diego, United States of America; ${ }^{2}$ Samumed, LLC, Chief Medical Officer, San Diego, United States of America

Background: Meniscal injuries are the most common pathology of the knee and are associated with pain, stiffness, and localized swelling. Menisca damage is a frequent finding on MRI images of knee osteoarthritis (OA). ${ }^{1}$ Efforts to repair meniscal damage have been largely unsuccessful and do not prevent the progression of degenerative changes that lead to knee OA. ${ }^{2}$ The Wnt signaling pathway has been shown to be regulated during meniscal development, ${ }^{3}$ suggesting that manipulation of this pathway may influence the regenerative capacity of the meniscus. Lorecivivint (LOR; SM04690) is an intra-articular (IA), small-molecule CLK2/DYRK1A inhibitor that modulates the Wnt pathway. ${ }^{4}$

Objectives: LOR was evaluated in preclinical studies to determine its protective and anabolic effects in ex vivo explants and in a rat model of chemically induced inflammatory meniscal degeneration.

Methods: Effects of LOR $(30 \mathrm{nM})$ on matrix metalloproteinase (MMP) expression in cultured rat menisci treated with IL-1B were measured by qRTPCR. In vivo, LOR activity was evaluated in a rat model of monosodium iodoacetate (MIA) injection-induced inflammatory meniscal degeneration. A single IA injection of MIA was immediately followed by a single IA injection of LOR $(0.3 \mathrm{ug})$ or vehicle. Knees were harvested on Days 1,4 , and 11 and menisci were isolated. Anti-inflammatory effects were evaluated by qRT-PCR for TNFA and IL6 expression. Meniscal protection was evaluated by qRTPCR for MMPs and aggrecanase. Anabolic effects were evaluated by qRTPCR for collagens.

Results: In ex vivo meniscal explants, LOR inhibited expression of MMP1, $M M P 3$, and MMP13 compared with DMSO $(P<0.01)$. In vivo, LOR significantly decreased expression of MMPs and aggrecanase $(P<0.05)$ and reduced expression of inflammatory cytokines TNFA and IL6 compared with vehicle in the rat model of inflammatory meniscal degeneration at Day 4 after MIA injection. Additionally, LOR increased expression of collagen types I, II, and III at Day 11 after MIA injection (Figure 1)

Conclusion: LOR exhibited protective effects in the meniscus ex vivo and in vivo by reducing catabolic enzyme expression compared with control. Anti-inflammatory effects of LOR were demonstrated by inhibition of inflammatory cytokine expression. Compared with vehicle, LOR increased collagen expression in vivo, indicating potential meniscal anabolic effects. These data support further investigation of LOR as a potential structure-modifying treatment for meniscal injuries. 